\title{
Systematic review and meta-analysis of the efficacy and safety of Biqi capsule in rheumatoid arthritis patients
}

\author{
XIU-MIN CHEN $^{1,2^{*}}$, JIA-QI WU ${ }^{3 *}$, QING-CHUN HUANG ${ }^{1}$, JIAN-YONG ZHANG $^{4}$, JIAN-HONG PEN ${ }^{5}$, \\ ZHI-SHENG HUANG ${ }^{6}$, YONG-LIANG CHU ${ }^{1}$, XIAO-HONG HE ${ }^{1}$, MAO-JIE WANG ${ }^{1}$ and RUN-YUE HUANG ${ }^{1}$ \\ ${ }^{1}$ Department of Rheumatology, The Second Affiliated Hospital, (Guangdong Provincial Hospital of Chinese Medicine), \\ Guangzhou University of Chinese Medicine; ${ }^{2}$ Postdoctoral Mobile Research Station; ${ }^{3}$ Second Clinical College, \\ Guangzhou University of Chinese Medicine, Guangzhou, Guangdong 510006; ${ }^{4}$ Department of Rheumatology, \\ Shenzhen Hospital of Chinese Medicine, Shenzhen, Guangdong 518033; ${ }^{5}$ Department of Rheumatology, \\ Dongguan Hospital of Chinese Medicine, Dongguan, Guangdong 523005; ${ }^{6}$ Department of Rheumatology, \\ Guangzhou Hospital of Chinese and Western Medicine, Huadu, Guangdong 510800, P.R. China
}

Received September 28, 2017; Accepted March 1, 2018

DOI: $10.3892 /$ etm.2018.6121

\begin{abstract}
Biqi capsule is a Traditional Chinese Medicine preparation for treating rheumatoid arthritis (RA), and clinical studies have indicatedthat its effect may be more beneficial than that of Western medicine. The present study aimed to estimate the efficacy and safety of Biqi capsule alone or combined with methotrexate (MTX) compared with MTX alone for treating RA by performing a meta-analysis of randomized controlled trials and controlled clinical trials. A systematic literature search of studies published until March 2017 was performed. References from relevant studies were screened to obtain additional articles. The results were independently evaluated for relevance, and full-text studies were assessed for eligibility. The risk of bias was assessed using the Cochrane collaboration tool for assessing risk of bias. Out of 558 citations that were initially retrieved, a total of 5 studies comprising 522 patients met the inclusion criteria. The risk of bias of these trials was generally unclear or high. Meta-analysis indicated that Biqi capsule had better effects on C-reactive protein [standardized mean difference (SMD), -7.05; 95\% CI -(10.77-3.33)] and tender joint count [SMD, -3.02; 95\% CI, -(3.81-2.22)] and fewer adverse effects (AEs) than MTX [relative risk (RR), 0.19;
\end{abstract}

Correspondence to: Dr Run-Yue Huang or Dr Qing-Chun Huang, Department of Rheumatology, The Second Affiliated Hospital, (Guangdong Provincial Hospital of Chinese Medicine), Guangzhou University of Chinese Medicine, 111 Dade Road, Yuexiu, Guangzhou, Guangdong 510006, P.R. China

E-mail: ry_huang@hotmail.com

E-mail: qch1963@163.com

${ }^{*}$ Contributed equally

Key words: rheumatoid arthritis, Biqi capsule, herbal medicine, systematic review, meta-analysis
95\% CI, 0.08-0.43]. Biqi capsule plus MTX was superior to MTX in terms of the total effect (RR, 1.17; 95\% CI, 1.06-1.28), rheumatoid factor [SMD, -12.54; 95\% CI, -(16.87-8.20)], swollen joint count [SMD, $-1.50 ; 95 \% \mathrm{CI},-(1.99-1.01)]$, score of joint swelling [SMD -2.07; 95\% CI, -(2.76-1.38)], tender joint count [SMD, -2.16; 95\% CI, -(2.86-1.47)] and score of joint tenderness [SMD, -4.69; 95\% CI, -(5.92-3.47)]. There was no difference in AEs between Biqi capsule plus MTX and MTX (RR, 0.71; 95\% CI, 0.34-1.50). In conclusion, the present study indicated that compared with MTX, Biqi capsule plus MTX appeared to have more benefits but that Biqi capsule alone was not better for RA patients than MTX. In the other words, Biqi capsule plus MTX is more effective and has fewer AEs compared to MTX. However, the trials selected in the present meta-analysis have various limitations, including the lack of blinding and the short duration of the treatment; therefore, the conclusions are not sufficiently definitive. More randomized controlled trials are necessary to evaluate the use of Biqi capsule for managing RA.

\section{Introduction}

Rheumatoid arthritis (RA) is a systemic inflammatory disorder whose prevalence is estimated to be $0.3-1.0 \%$ worldwide $(1,2)$. As a refractory disease, RA leads to not only a poor quality of life for patients, but also a heavy burden on society and the economy (3). In general, the drugs used for RA therapy include corticosteroids, non-steroidal anti-inflammatory drugs, conventional disease-modifying antirheumatic drugs (cDMARDs) and biological DMARDs. Of note, as a typical DMARD, methotrexate (MTX) is recommended as a first-line drug for the management of early and established RA (4).

On occasion, cDMARDsdo notdecisively control the disease activity of RA (5). In addition, the long-term use of cDMARDs results in a reduction of drug efficacy and an accumulation of side effects (6). Biological agents, includingtumor necrosis factor (TNF)- $\alpha$ inhibitor, work faster in reducing disease activity than cDMARDs, but certain patients 
fail to maintain an initial response due to acquired drug resistance (7). Therefore, it is a concern for rheumatologists to optimize the current therapeutic strategies for RA management. Furthermore, in patients with MTX-refractory early RA, a treatment strategy commencing with the addition of the TNF inhibitor infliximab over 21 months has been associated with similar effects to those of salazosulfapyridine and hydroxychloroquine but higher costs (8). In fact, the use of biological agents imposes a heavy economic cost on the patients and on society in China $(3,9)$.

Traditional Chinese Medicine (TCM) providesvarious useful and inexpensive methods for RA therapy, and TCM is frequently used as an add-on therapy in China. In addition, TCM is generally safer than cDMARDs (10). For instance, recently, in a 24-week, randomized, multicenter, single-blinded study, managing RA by using TCM appeared to be effective for treating RA and was associated with fewer side effects compared with cDMARDs (10). In the present study, clinical responses were defined by the American College of Rheumatology (ACR) core set of disease activity measures (11). An ACR20/50/70 response was defined as at least 20/50/70\% improvement in the tender joint count and the swollen joint count and at least 20/50/70\% improvement in 3 of the 5 core set measures, including the physician's assessment of disease activity, the patient's assessment of disease activity, the patient's assessment of pain, the patient's assessment of physical function and the levels of an acute-phase reactant [either the $\mathrm{C}$-reactive protein (CRP) levels or the erythrocyte sedimentation rate (ESR)] (11). Although the ACR 20 and ACR50 responses at 24 weeks were better in the group receiving cDMARD treatment, the ACR 70 response was not significantly different between the TCM and cDMARD groups, suggesting that the long-term use of TCM may have a similar clinical outcome to the long-term use of cDMARDs (10).

Biqi capsule is a TCM preparation for treating RA. It is composed of the followingnatural materials: The dried fruit of Strychnosnux-vomica (Maqianzi), the dried body of Pheretima aspergillum (Dilong), the root of Codonopsispilosula (Dangshen), the sclerotia of Poriacocos (Fuling), the rhizoma of Atractylodismacrocephalae (Baizhu), the root and rhizoma of Glycyrrhizauralensis (Gancao), the rhizoma of Ligusticumwallichii (Chuanxiong), the root and rhizoma of Salvia miltiorrhiza (Danshen), the root and rhizoma of Panaxnotoginseng (Sanqi) and the radix of Achyranthesbidentata (Niuxi). The dried fruit of Strychnosnux-vomicais the most important component of Biqi capsule. It has been proved that processing of semen strychnimay reduce the level of nitric oxide (NO) and NO synthase (NOS) in serum of rats, thereby possibly regulating immune disorders associated with RA (12). In addition, it has been demonstrated that cryptotanshinone, brucine and strychnine are the major anti-inflammatory components in the aqueous extract of Biqi capsule (13). Cryptotanshinone not only inhibits the secretion of NO but also suppresses the secretion of interleukin (IL)-6 in lipopolysaccharide (LPS)-induced RAW 264.7 cells (13).

Brucine, a major alkaloid monomer from the dried fruit of Strychnosnux-vomica, relieves pain, reduces inflammation, regulates cytokine expression and inhibits the proliferation of synovial fibroblasts; therefore, it has been frequently used to treat RA (14). Similar to numerous other TCM formulations, Biqi capsule has long been used to manage RA. It has been demonstrated that Biqi capsule effectively improves the clinical signs and symptoms of RA (15) and has the effect of relieving pain, eliminating inflammation in adjuvant arthritis (16) and regulating immune responses in RA (17). Pre-clinical studies have also demonstrated that Biqi capsule decreases lipid peroxidation and inflammatory responses induced by lipopolysaccharide in articular chondrocytes, enhances superoxide dismutase (SOD) activity of chondrocytes and oxyradical clearance, and protects the chondrocytes against LPS-induced inflammatory damage (18). However, the effect and safety of Biqi capsule in the treatment of RA and whether it is as effective as cDMARDsremain controversial. In the present study, a meta-analysis of randomized controlled trials (RCTs) and controlled clinical trials (CCTs) on RA patients was performed to assess the efficacy and safety of Biqi capsule alone or combined with MTX vs. MTX.

\section{Materials and methods}

Inclusion criteria. The present systematic review and meta-analysis wereprepared according to the Preferred Reporting Items for Systematic Reviews and Meta-Analyses statement (19).

Patients in the studies involved were diagnosed with RA according to the criteria of the 1987 classification of the American College of Rheumatology (ACR) (20). All RCTs and CCTs including the comparison of Biqi capsule alone or combined with MTX and MTX in treating RA were considered. There were no restrictions for sex, age, disease course or administration doses of MTX. Multiple publications reporting the same groups of participants were excluded. Biqi capsule alone or combined with MTX was regarded as the experimental treatment and the cohort treated with MTX was regarded as a control.

Outcomes. The following outcomes were recorded: Clinical efficacy, adverse events (AEs), tender joint count (TJC), swollen joint count (SJC), score of joint tenderness (JTS), score of joint swelling (JSS), changes in rheumatoid factors (RF), CRP and ESR. The outcomes that were only sporadically reported were eliminated from the final analysis. The number of patients treated effectively in each group was counted based on the categories of 'cured', 'markedly effective' and 'effective'. Patients who were cured were defined as those having four out of the five core set measures, including morning stiffness that lasted $<15 \mathrm{~min}$, no fatigue, no painful and tender joints, no SJC, and an ESR $<20 \mathrm{~mm} / \mathrm{h}$ (male) or $30 \mathrm{~mm} / \mathrm{h}$ (female). Patients in which the treatment was markedly effective were defined as those having four of the six core set measures, including $\geq 50 \%$ decrease in the duration of morning stiffness, TJC, SJC, ESR or CRP, $\geq 50 \%$ increase ingrip strength, a $>2$-fold decrease in RF. Patients in which the treatment was markedly effective were defined as those havingthree of the aforementionedsix core set measures (15). The overall efficacy determined as the proportion of these patients compared with the total number of patients. Continuous data (RF, CRP, ESR, TJC, SJC, JTS and JSS) were expressed as the mean with standard deviation 
and dichotomous variables (the clinical efficacy and AEs) were expressed as absolute numbers for further statistical analysis.

Search methods. Associated studies from inception to March 2017 were retrieved from the following databases: Medline/PubMed, EmBase, the Cochrane Library, the China National Knowledge Infrastructure, the Chinese Medical Journal Database, Wang fang Data, VIP journal integration platform, the Chinese Biomedical Literature Database, the Chinese Scientific and Technological Journals Database, the Traditional Chinese Medicine Database and the China Doctorate Dissertation Full Text Database. The search terms included 'Biqi capsule' or 'Biqi' combined with 'rheumatoid arthritis', 'arthritis, rheumatoid' or 'RA'. Furthermore, the references of selected articles were carefully screened, so that any studies fulfilling the inclusion criteria of the present study could be analyzed. Furthermore, recent data were manually retrieved by searching the periodicals, journals and symposium abstracts published until March 2017 that were available at the library of Guangzhou University of Chinese Medicine (Guangzhou, China). The language was limited to English or Chinese. The search was independently performed by three authors (XC, JW and RH).

Data extraction and handling of incomplete data. Trial selection and data extraction were performed by three authors (XC, JW and $\mathrm{RH}$ ), and the relevant data were extracted andentered into an Excel spreadsheet. Two review authors (XC and JW) screened all selected articles and extracted the data independently. The relevant data included characteristics of trials, the first author, year of publication, baseline characteristics of patients, number of patients in the experimental group (EG) and control group (CG), duration of the study, intervention (treatment) methods, outcome measures and mainresults. Disagreements were resolved by discussion with the third review author (RH) and a group of rheumatologists (QH, MQ, YC and XH).

Risk of bias in individual studies. The risk of bias of the trials included in the present review was assessed using the Cochrane collaboration's tool for assessing the risk of bias by two review authors (XC and JW) independently (21). Seven different risk of bias domains were assessed at the study level: Sequence generation, allocation concealment, study blinding, outcome assessor blinding, incomplete outcome data, selective outcome reporting and other bias. Each of the 7 specified risk of bias domains was evaluated in 3 groups: Low-risk, high-risk or unclear risk (22).

Statistical analysis. Review Manager software, version 5.3. (The Cochrane Collaboration, London, UK), was used to analyze the clinical data collected. The dichotomous outcomes, including treatment effects and AEs, were evaluated using the relative risk (RR) with the $95 \%$ confidence interval (CI). The standardized mean differences (SMDs) were calculated by dividing the difference between experimental groups and controls by the baseline variance. The SMD was used when different scales were used to measure the same concept. Sensitivity analyses were also used in this study. Heterogeneity

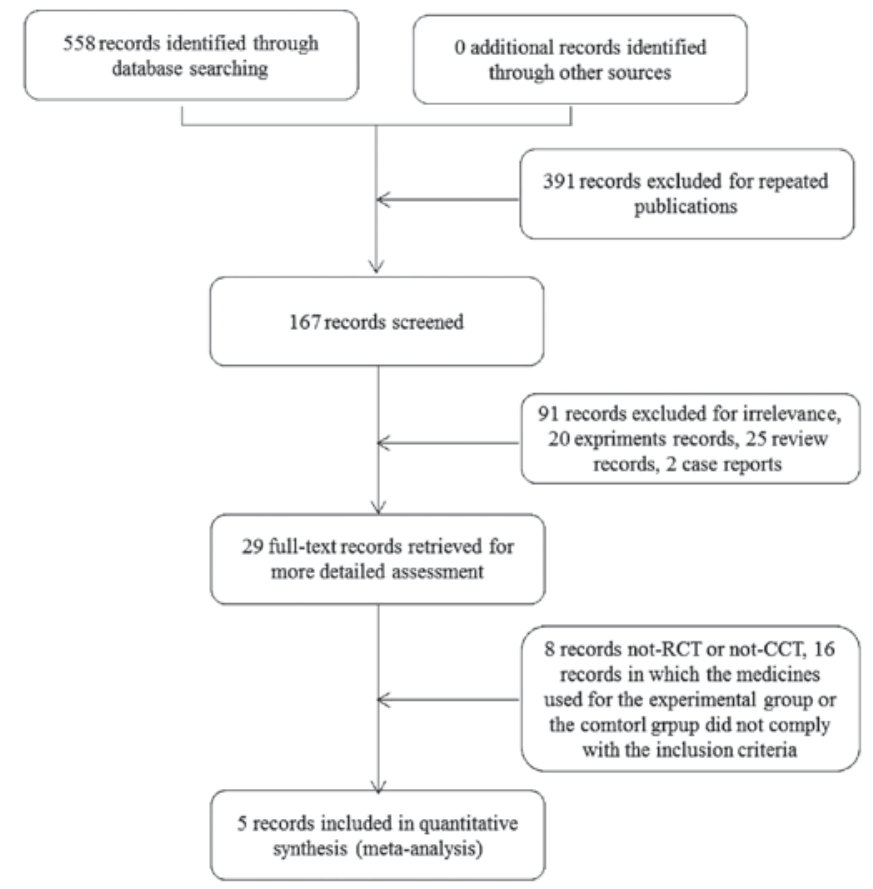

Figure 1. Flow chart of the search strategy. RCT, randomized controlled trial; CCT, controlled clinical trial.

was tested and was considered significant when $\mathrm{I}^{2}$ was $>50 \%$. A random-effects model was used for the meta-analysis if significant heterogeneity existed, and a fixed-effects model was used when the heterogeneity was not significant. If the data provided by the included studies were not appropriate for performing a meta-analysis, the study data were presented in narrative form.

\section{Results}

Search results and description of selected studies. From an initially retrieved 558 records, a total of 5 RCTs were identified (23-27), including 522 patients that matched the inclusion criteria (Fig. 1). The details of these trials extracted in the present study are summarized in Table I. All 5 trials had been performed in China and published in Chinese from 2004 to 2016. The manufacturer of Biqi capsule in these 5 trials was identical (Tianjin DarentangJingwanhong Pharmaceutical Co. Ltd., Tianjin, China), so that the quality control of Biqi capsules was ensured and its composition was identical amongst all studies included. The 5 trials were non-blinded studies. The interventions included Biqi capsule or Biqi capsule plus MTX. The group treated with MTX only was considered to be the control group. Of the trials included, 3 compared Biqi capsule with MTX (23-25), and 3 compared Biqi capsule plus MTX with MTX (25-27). The duration of treatment in the 5 trials was 8-12 weeks, how ever the different durations of treatment were notcompared during the meta-analysis.

Risk of bias in the included studies. Fig. 2 presents a summary of the risk of bias in the 5 trials included in the present review. The risk of bias was considered to be high in all trials. Two studies did not mention a random method $(23,27)$. Allocation 
Table I. Characteristics of individual studies.

\begin{tabular}{|c|c|c|c|c|c|c|c|}
\hline \multirow[b]{2}{*}{ Author, year } & \multicolumn{2}{|c|}{$\begin{array}{l}\text { Sample } \\
\text { size }\end{array}$} & \multicolumn{2}{|c|}{$\begin{array}{l}\text { Intervention } \\
\text { method }\end{array}$} & \multirow{2}{*}{$\begin{array}{l}\text { Duration } \\
\text { (weeks) }\end{array}$} & \multirow[b]{2}{*}{ Outcomes } & \multirow[b]{2}{*}{ (Refs.) } \\
\hline & EG & CG & EG & $\mathrm{CG}$ & & & \\
\hline Liu et al, 2005 & 60 & 60 & BQC $1.2 \mathrm{~g}$ tid & MTX 15 mg qw & 12 & TE, RF, ESR, AE, TJC, SJC, & $(23)$ \\
\hline Bai and $\mathrm{Li}, 2009$ & 50 & 18 & $\mathrm{BQC} 1.2 \mathrm{~g}$ tid & MTX 10 mg qw & 8 & TE, CRP, ESR, AE, JTS, JSS & (24) \\
\hline Jie et al, 2012 & 44 & 46 & BQC $1.2 \mathrm{~g}$ tid & MTX 10 mg qw & 12 & TE, RF, ESR, CRP, TJC, SJC, JTS, JSS & $(25)$ \\
\hline Wan et al, 2012 & 26 & 26 & $\begin{array}{l}\text { BQC } 1.2 \mathrm{~g} \mathrm{tid} \\
\text { MTX } 7.5 \mathrm{mg} \text { qw }\end{array}$ & MTX $7.5 \mathrm{mg} \mathrm{qw}$ & 12 & TE, RF, AE, TJC, SJC, JTS, JSS & $(26)$ \\
\hline Rao et al, 2016 & 72 & 72 & $\begin{array}{l}\text { BQC } 1.2 \mathrm{~g} \mathrm{tid}, \\
\text { MTX } 10 \mathrm{mg} \mathrm{qw}\end{array}$ & MTX 10 mg qw & 12 & TE, AE, TJC, SJC, JTS, JSS & (27) \\
\hline Jie et al, 2012 & 48 & 46 & $\begin{array}{l}\text { BQC } 1.2 \mathrm{~g} \mathrm{tid}, \\
\text { MTX } 10 \mathrm{mg} \mathrm{qw}\end{array}$ & MTX 10 mg qw & 12 & TE, RF, ESR, CRP, TJC, SJC, JTS, JSS & $(25)$ \\
\hline
\end{tabular}

BQC, Biqi capsule; MTX, methotrexate; EG, experimental group; CG, control group; TE, total effect; RF, rheumatoid factor; ESR, erythrocyte sedimentation rate; CRP, C-reactive protein; AE, adverse effect; TJC, tender joint count; SJC, swollen joint count; JTS, score of joint tenderness; JSS, score of joint swelling; tid, three times per day; qw, once weekly.

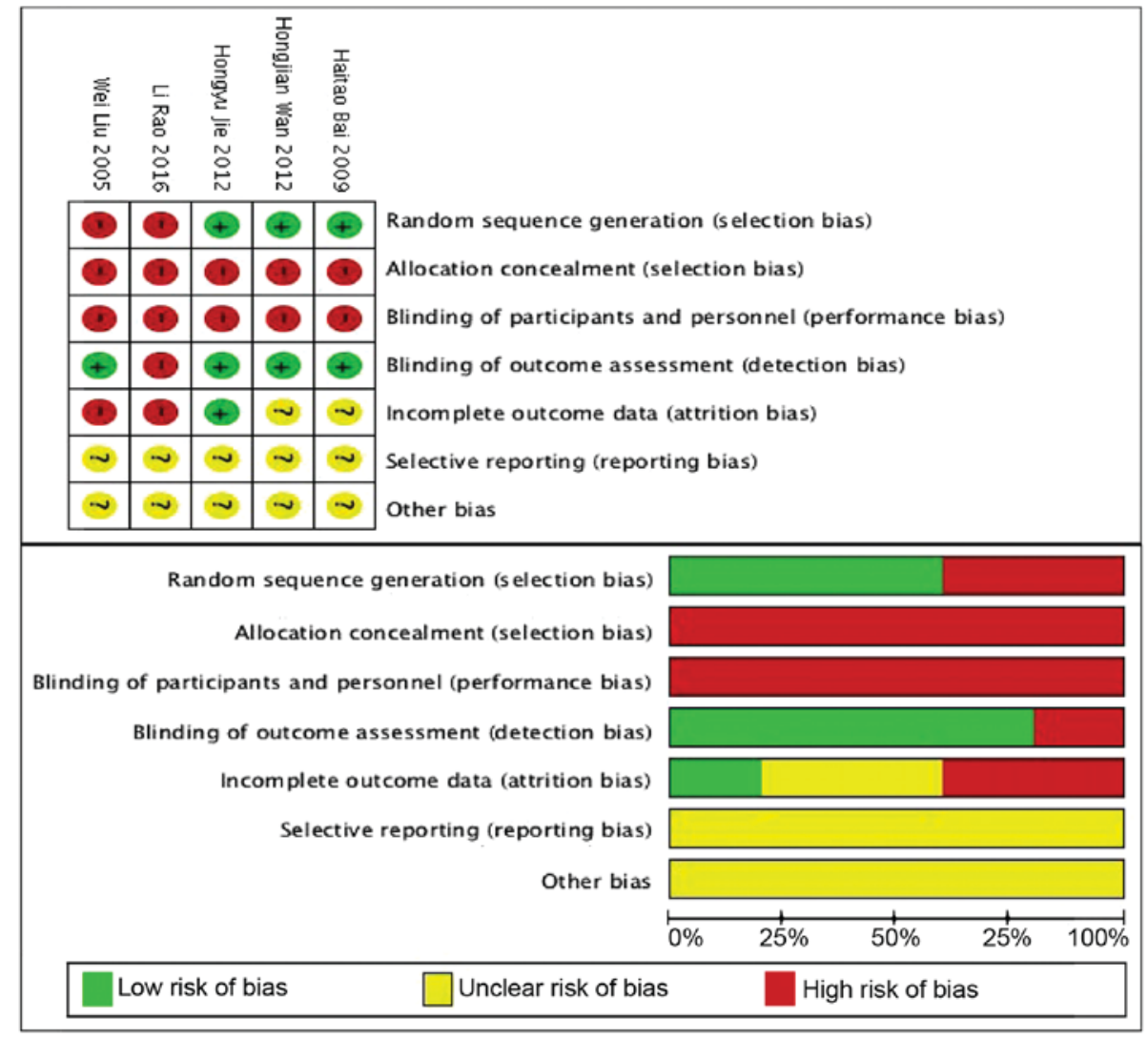

Figure 2. Risk of bias summary and risk of bias graph.

concealment and blinding were poorly reported. Regarding incomplete data, which is attrition bias, only one study was considered to have a low risk of bias (25), and the other 4 studies hadan unclear or high risk. In terms of selective reporting and other biases, all studies were considered to have an unclear risk.
Overall efficacy. All 5 trials included in the present review provided the overall efficacy of Biqialone or plus MTX compared with MTX. Fig. 3A demonstrated that $\mathrm{I}^{2}=73 \%$ $(\mathrm{P}=0.02)$, therefore the analysis used a random effects model. The results indicated that no difference was identified between Biqi capsule and MTX (RR, 1.35; 95\% CI, 1.00-1.82; P=0.05). 


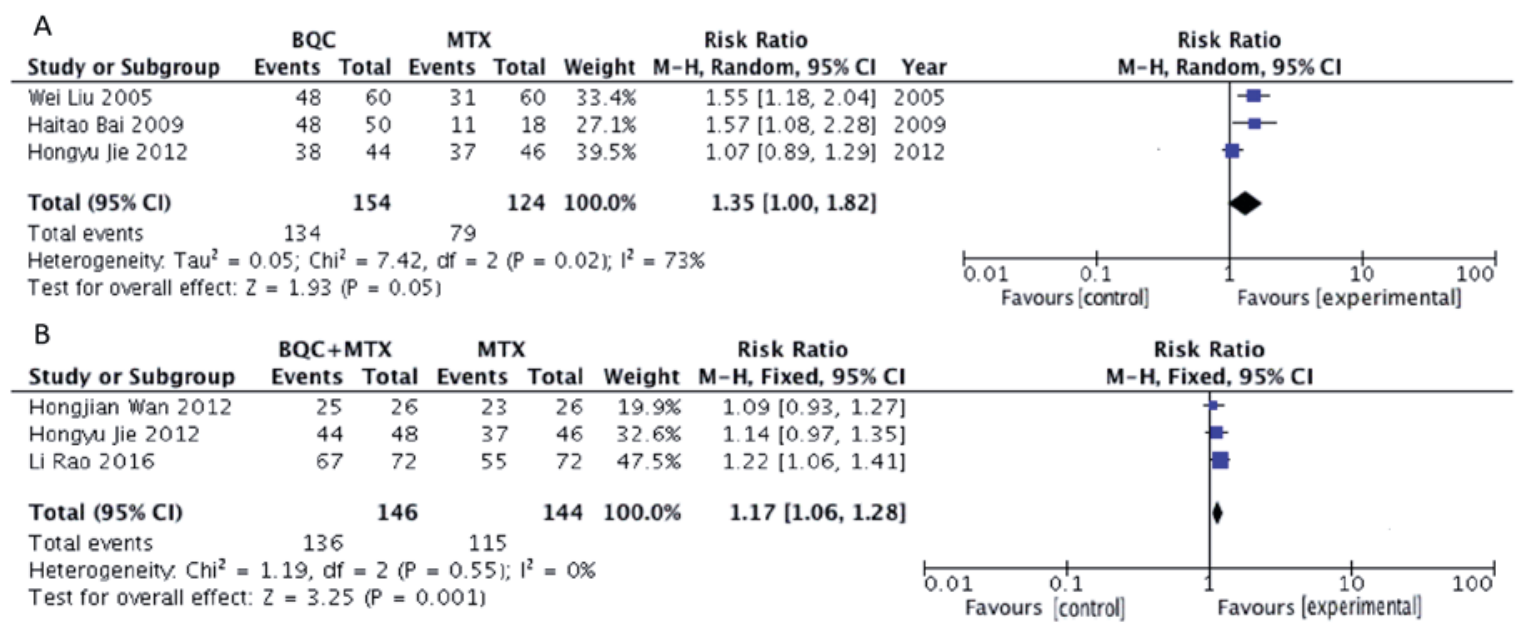

Figure 3. Analysis of the overall efficacy of Biqi capsule or Biqi capsule plus MTX vs. MTX in the treatment of rheumatoid arthritis. (A) Overall efficacy of Biqi capsule compared with MTX using a random-effects model. (B) Overall efficacy of Biqi capsule plus MTX compared with MTX using a fixed-effects model. The $\mathrm{I}^{2}$ and $\mathrm{P}$-value were regarded as criteria of heterogeneity. The blue squares indicate the relative risk and their $95 \% \mathrm{CI}$. The black diamonds indicate the pooled relative risk and 95\% CI. CI, confidence interval; BQC, Biqi capsule; MTX, methotrexate; M-H, Mantel-Haentzel; df, degrees of freedom.

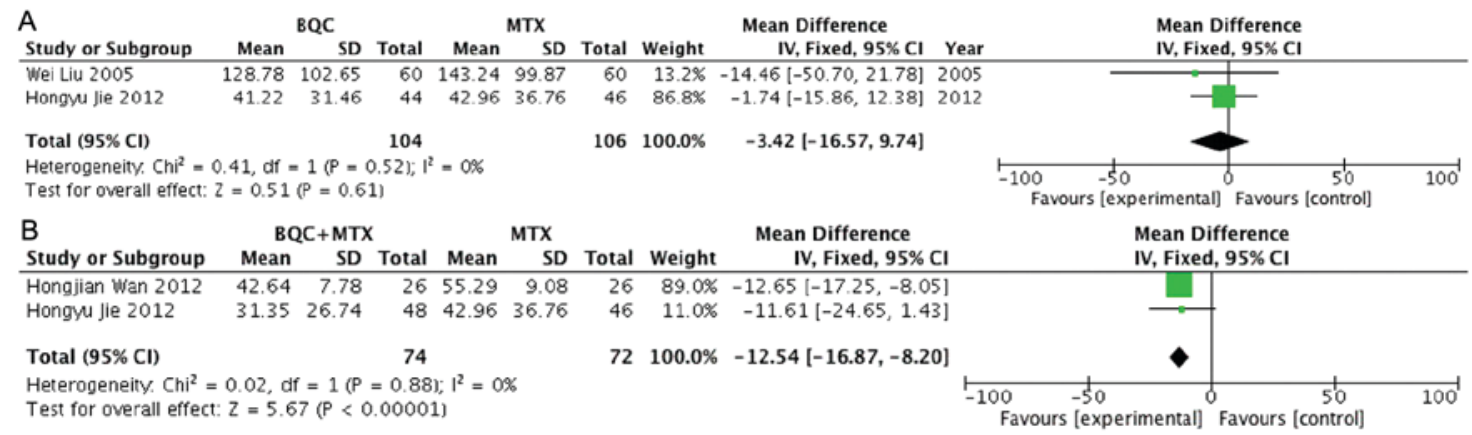

Figure 4. Analysis of the effects of Biqi capsule or Biqi capsule plus MTX vs. MTX on RF in patients with rheumatoid arthritis. (A) RF of Biqi capsule compared with MTX using a fixed-effects model. (B) RF of Biqi capsule plus MTX compared with MTX using a fixed-effects model. The I ${ }^{2}$ and P-value were regarded as criteria of heterogeneity. The green squares indicate the relative risk and their $95 \%$ CI. The black diamonds indicate the pooled relative risk and 95\% CI. CI, confidence interval; BQC, Biqi capsule; MTX, methotrexate; df, degrees of freedom; IV, inverse variance; SD, standard deviation; RF, rheumatoid factors.

Biqi capsule and MTXhad similar overall efficacy. Furthermore, Fig. 3B revealed that $\mathrm{I}^{2}=0 \%(\mathrm{P}=0.55)$, so the analysis used a fixed effects model. A significant difference was observed in the number of patients who were treated effectively by Biqi capsule plus MTX vs. MTX, (RR, 1.17; 95\% CI, 1.06-1.28; Fig. 3).

Laboratory indexes. Of the 5 trials, 3 included data on the serum concentration of RF. Fig. 4 demonstrated that there was no heterogeneity, therefore the analysis was performed using a fixed effects model. Meta-analysis indicated no significant difference between Biqi capsule and MTX (SMD, -3.42; $95 \%$ CI, -16.57-9.74) but a significant difference between Biqi capsule plus MTX and MTX [SMD, -12.54; 95\% CI, -(16.87-8.20); Fig. 4]. Three trials were included in the meta-analysis on the ESR. Fig. 5 revealed that $\mathrm{I}^{2}=0 \%$, therefore afixed effect model was adopted. A statistically significant difference in the reduction of the ESR was identified between Biqi capsule and MTX [SMD, -4.74; 95\% CI, -(9.10-0.38); Fig. 5]. Among the trials selected, 2 studies provided data on CRP. As illustrated in Fig. 6, $\mathrm{I}^{2}=0 \%$, and a fixed effects model was adopted for the analysis. The combined SMD was -7.05, and the $95 \%$ CI ranged from -10.77 to $-3.33(\mathrm{P}=0.0002)$, which indicated a significant difference between Biqi capsule and MTX (Fig. 6). However, it was not possible to perform any meta-analysis for the comparison between Biqi capsule plus MTX and MTX in reducing ESR or CRP, as only one or none of the studiesreported on them. Overall, it may be concluded thatregarding the capacity to reduce the serum RF concentration in RA patients, no difference was identified between Biqi capsule alone and MTX, but a significant difference was determined between Biqi capsule plus MTX and MTX. Biqi capsule plus MTX was more efficacious on reducing the serum RF concentration compared with MTX. Regarding the capacity to reduce the serum ESR and CRP levels, Biqi capsule was more efficacious compared with MTX.

Improvement of clinical signs and symptoms. A total of 4 trials compared TJC and SJC between different treatment arms. As illustrated in Fig. 7, $\mathrm{I}^{2}=0 \%$ or $43 \%$, there fore a fixed effects model was adopted for the analysis. The meta-analysis indicated a significant difference between Biqi capsule and MTX [SMD, -3.02; 95\% CI, -(3.81-2.22)] 


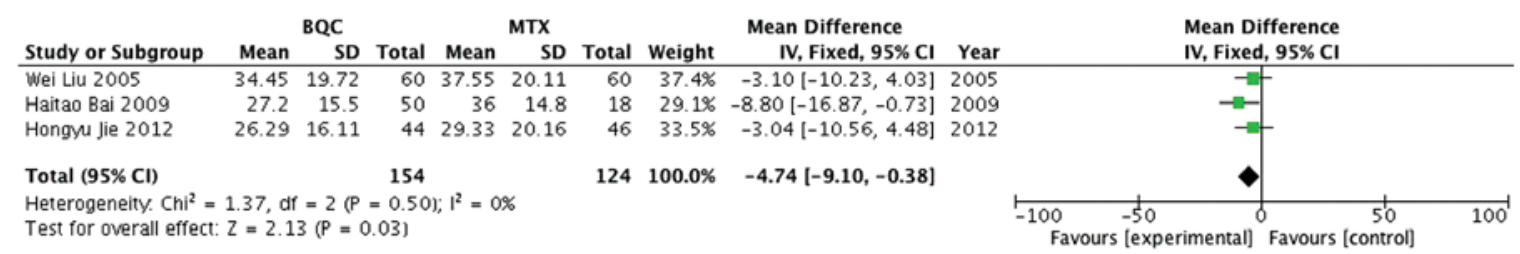

Figure 5. Analysis of the effect of Biqi capsule vs. MTX on the erythrocyte sedimentation rate in rheumatoid arthritis patients. The $\mathrm{I}^{2}$ and P-value were regarded as criteria of heterogeneity. The green squares indicate the relative risk and their $95 \%$ CI. The black diamonds indicate the pooled relative risk and 95\% CI. CI, confidence interval; BQC, Biqi capsule; MTX, methotrexate; df, degrees of freedom; IV, inverse variance; SD, standard deviation.

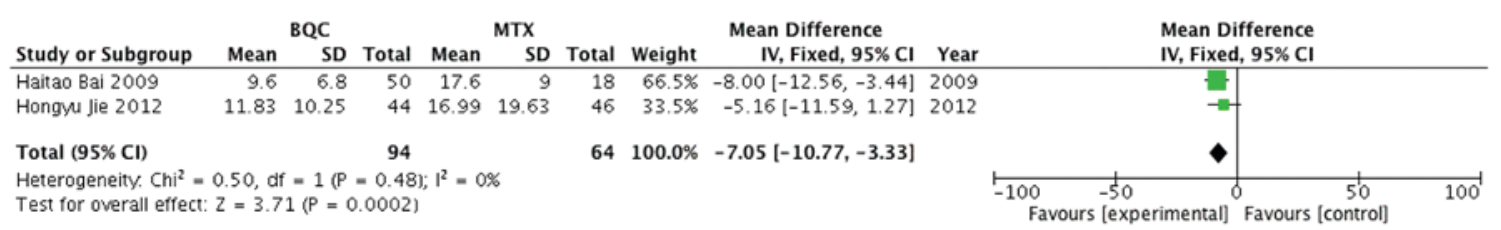

Figure 6. Analysis of the effect of Biqi capsule vs. MTX on C-reactive protein in patients with rheumatoid arthritis. The $\mathrm{I}^{2}$ and P-value were regarded as criteria of heterogeneity. The green squares indicate the relative risk and their 95\% CI. The black diamonds indicate the pooled relative risk and $95 \% \mathrm{CI}$. CI, confidence interval; BQC, Biqi capsule; MTX, methotrexate; df, degrees of freedom; IV, inverse variance; SD, standard deviation.

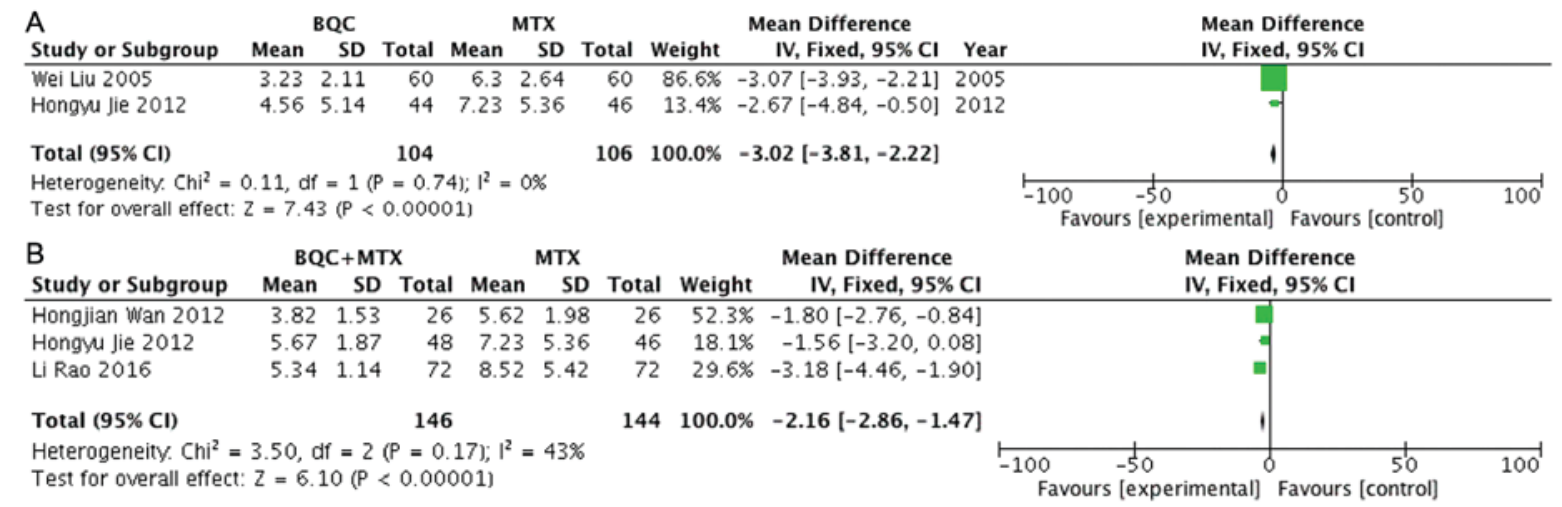

Figure 7. Analysis of the TJC in rheumatoid arthritis patients treated with Biqi capsule or Biqi capsule plus MTX. (A) TJC in patients treated with Biqi capsule vs. MTX using a fixed-effects model. (B) TJC in patients treated with Biqi capsule plus MTX vs. MTX using a fixed-effects model. The $\mathrm{I}^{2}$ and P-value were regarded as criteria of heterogeneity. The green squares indicate the relative risk and their 95\% CI. The black diamonds indicate the pooled relative risk and 95\% CI. CI, confidence interval; BQC, Biqi capsule; MTX, methotrexate; df, degrees of freedom; IV, inverse variance; SD, standard deviation; TJC, tender joint count.

or Biqi capsule plus MTX and MTX [SMD, $-2.16 ; 95 \%$ CI, $-(2.86-1.47)]$ in terms of TJC. Fig. 8A demonstrated that $\mathrm{I}^{2}=95 \%$, therefore the analysis used a random effects model. Fig. 8B revealed that there was no heterogeneity, therefore a fixed effects model was used. Regarding the effect on the SJC, the meta-analysis indicated no significant difference between Biqi capsule and MTX (SMD, -0.90; 95\% CI, -5.02-3.22) but a significant difference between Biqi capsule plus MTX and MTX [SMD, -1.50; 95\% CI, -(1.99-1.01); Fig. 8]. The JTS and JSS after treatment were provided by 4 trials. As illustrated in Figs. 9 and 10 , when $\mathrm{I}^{2}<50 \%$, a fixed effects model was adopted, while if $\mathrm{I}^{2}>50 \%$, a random effects model was used. The results indicated no difference between Biqi capsule and MTX (JTS: SMD, -4.31 ; 95\% CI, -9.47-0.85; JSS: SMD, -0.39; 95\% CI, -3.52-2.74) but a significant difference between Biqi capsule plus MTX and MTX [JTS: SMD, -4.69; 95\% CI, -(5.92-3.47); JSS: SMD, $-2.07,95 \%$ CI, -(2.76-1.38); Figs. 9 and 10]. In summary, the results indicated that Biqi capsule alone or plus MTX may decrease TJC better compared withMTX. At the same time, on amelioration of SJC, JTS and JSS, Biqi capsule plus MTX was more efficacious compared with MTX.

AEs. AEs were described in 4 of the trials included. The most common AEs in the Biqi capsule groups were elevated blood pressure, gastrointestinal discomfort, skin discomfort and dizziness, and in the control groups, they were gastrointestinal discomfort, abnormal liver function and blood cell reduction. None of these AEs affected the treatment of RA whether or not AEs occurred. The meta-analysis demonstrated that there was no heterogeneity, and the analysiswas performed using a fixed effects model. According to the meta-analysis, a statistically significant difference was presentbetween the AEs in the groups treated with Biqi capsule and those with MTX (RR, 0.19; 95\% CI, 0.08-0.43), but no significant difference was observed between Biqi capsule plus MTX and MTX (RR, 0.71; 95\% CI, 0.34-1.50; Fig. 11). There were fewer AEs when using Biqi capsule to treat RA compared with the use of MTX. 


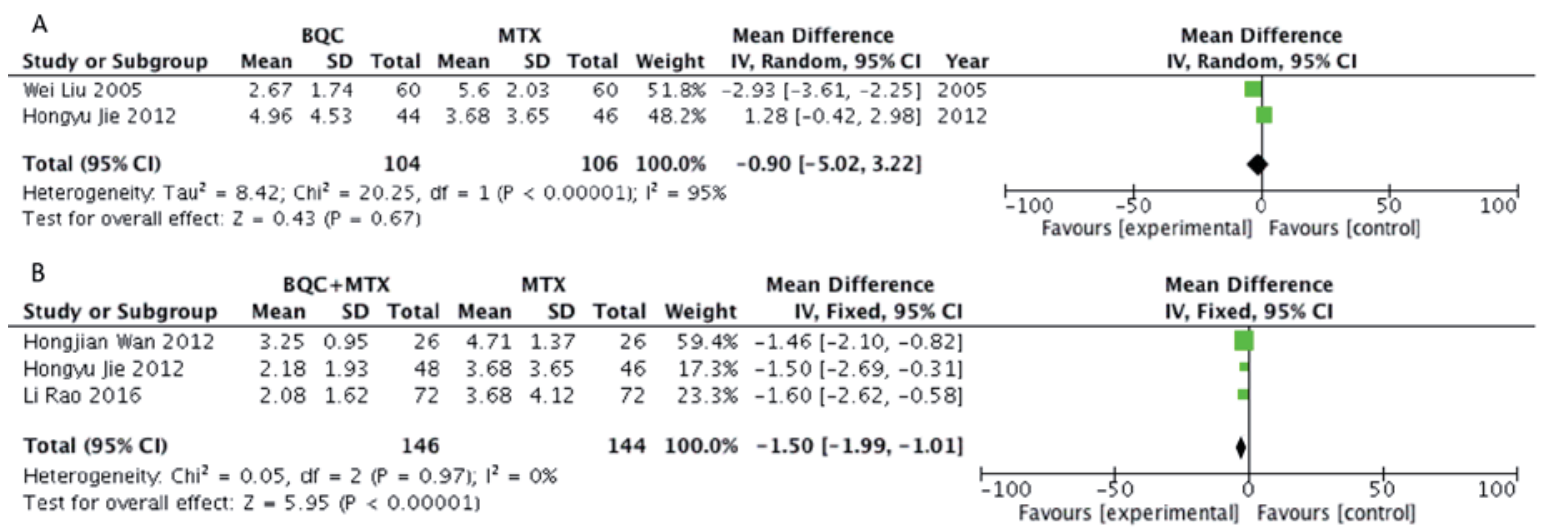

Figure 8. Analysis of SJC in rheumatic arthritis patients treated with Biqi capsule or Biqi capsule plus MTX. (A) SJC in patients treated with Biqi capsule compared with MTX using a random-effects model. (B) SJC in patients treated with Biqi capsule plus MTX compared to MTX using a fixed-effects model. The $\mathrm{I}^{2}$ and $\mathrm{P}$-value were regarded as criteria of heterogeneity. The green squares indicate the relative risk and their $95 \%$ CI. The black diamonds indicate the pooled relative risk and 95\% CI. CI, confidence interval; BQC, Biqi capsule; MTX, methotrexate; df, degrees of freedom; IV, inverse variance; SD, standard deviation; SJC, swollen joint count.

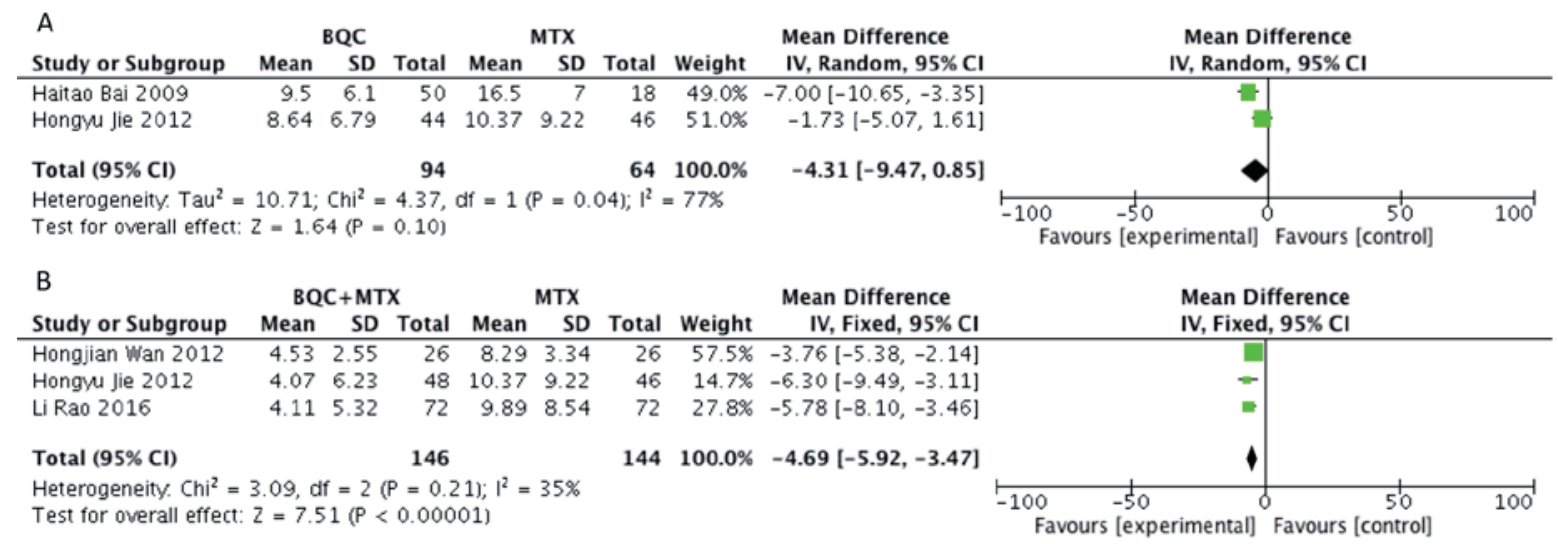

Figure 9. Analysis of JTS in rheumatoid arthritis patients treated with Biqi capsule or Biqi capsule plus MTX. (A) JTS in patients treated with Biqi capsule compared with MTX using a random-effects model. (B) JTS in patients treated with Biqi capsule plus MTX compared with MTX using a fixed-effects model. The $\mathrm{I}^{2}$ and P-value were regarded as criteria of heterogeneity. The green squares indicate the relative risk and their 95\% CI. The black diamonds indicate the pooled relative risk and 95\% CI. CI, confidence interval; BQC, Biqi capsule; MTX, methotrexate; df, degrees of freedom; IV, inverse variance; SD, standard deviation; JTS, score of joint tenderness.

\section{Discussion}

Biqi capsule, which is a typical TCM preparation for the treatment of RA, has been demonstrated to be effective and safe in the treatment of RA. Studies have indicated that Biqi capsule improves clinical symptoms and reduces inflammatory markers in RA patients $(28,29)$. Indeed, as supported by the effects of the major components, numerous in vivo studies have indicated that Biqi capsule significantly reduces the swelling degree of ankles, synovial hyperplasia, pannus formation, and the destruction of articular cartilage and bone $(18,30,31)$. The possible mechanisms have also been demonstrated in animal models of RA, and Biqi capsule is thought to exert its effects by reducing the expression of Janus kinase 3 and signal transducer and activator of transcription 3 , and by suppressing the expression of interleukin (IL)-4, interferon- $\gamma$, IL-1 and other inflammatory cytokines (30), as confirmed by several clinical studies reporting that Biqi capsule markedly decreases the levels of IL-17, IL-1 and TNF- $\alpha$ in patients with RA $(23,26)$. Of note, it was reported that the decreases in IL-1 and TNF- $\alpha$ were accompanied by an improvement in the clinical symptoms and physical signs of patients with RA (23). Taken together, these observations strongly suggest that Biqi capsule improvesaffected joints and systemic pathological changes in RA and inhibits joint damage in patients with RA, which is in agreement with the results of the present study.

The meta-analysis performed in the present study indicated that, compared with MTX, Biqi capsule may be more favourable with regard to reducing serum of CRP and decreasing the TJC, and may possess an efficacy comparable to that of MTX for ameliorating the serum levels of ESR. In addition, Biqi capsule plus MTX appeared to have a higher clinical efficacy than MTX, and in a significantly higher proportion of RA patients treated with Biqi capsule plus MTX, the clinical condition wasmarkedly improved after treatment. Furthermore, combination treatment may be better than MTX alone for the amelioration of RF, TJC, SJC, JTS and JSS

No severe side effects were reported in any of the included trials. MTX, as a first-line drug for treating RA, may cause a 


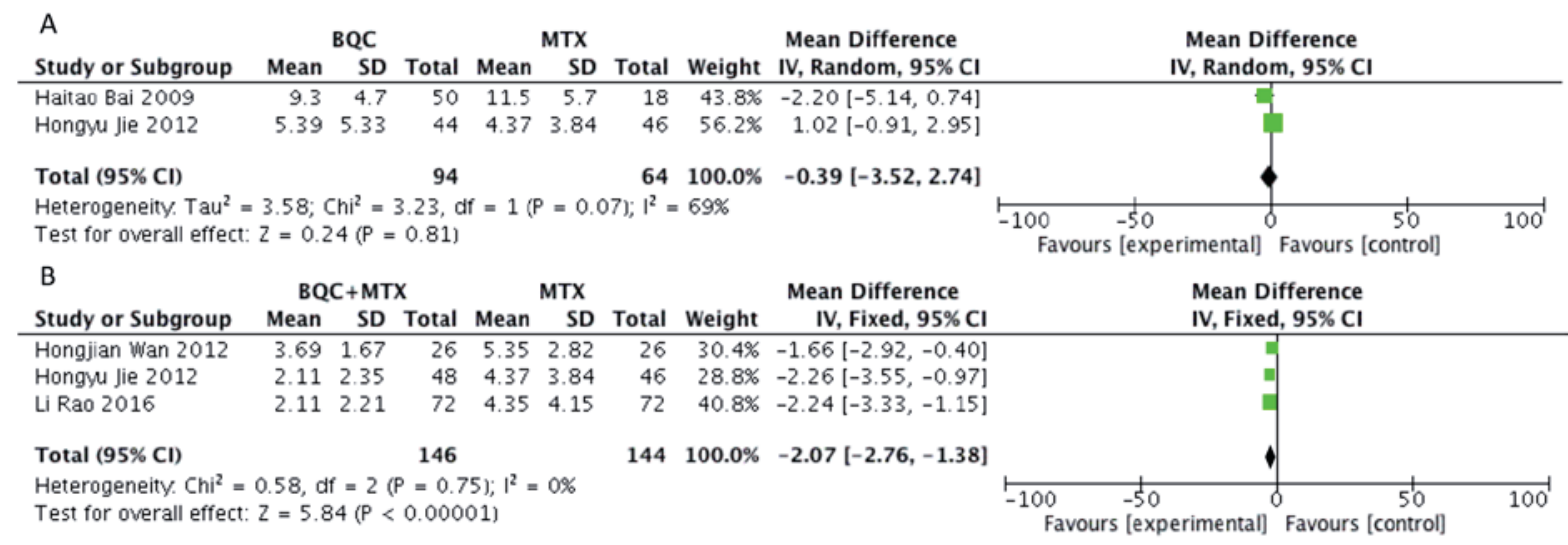

Figure 10. Analysis of JSS in rheumatoid arthritis patients treated with Biqi capsule or Biqi capsule plus MTX. (A) JSS in patients treated with Biqi capsule compared with MTX using a random-effects model. (B) JSS in patients treated with Biqi capsule plus MTX compared with MTX using a fixed-effects model. The $\mathrm{I}^{2}$ and $\mathrm{P}$-value were regarded as criteria of heterogeneity. The green squares indicate the relative risk and their 95\% CI. The black diamonds indicate the pooled relative risk and 95\% CI. CI, confidence interval; BQC, Biqi capsule; MTX, methotrexate; df, degrees of freedom; IV, inverse variance; SD, standard deviation; JSS, score of joint swelling.

\begin{tabular}{|c|c|c|c|c|c|c|c|c|c|}
\hline \multirow{2}{*}{$\begin{array}{l}\text { A } \\
\text { Study or Subgroup } \\
\text { Wei Liu } 2005\end{array}$} & \multicolumn{2}{|c|}{$\begin{array}{c}\text { BQC } \\
\text { Events Total }\end{array}$} & MTX & & \multicolumn{2}{|r|}{ Risk Ratio } & \multicolumn{3}{|c|}{$\begin{array}{c}\text { Risk Ratio } \\
\text { M-H, Fixed, } 95 \% \mathrm{CI} \\
\end{array}$} \\
\hline & 3 & 60 & 10 & 602 & $40.5 \%$ & $0.30[0.09,1.04] 2005$ & & & \\
\hline Haitao Bai 2009 & 3 & 50 & 10 & 185 & $59.5 \%$ & $0.11[0.03,0.35] 2009$ & & & \\
\hline Total $(95 \% \mathrm{Cl})$ & & 110 & & $78 \quad 10$ & $100.0 \%$ & $0.19[0.08,0.43]$ & & & \\
\hline \multirow{2}{*}{\multicolumn{7}{|c|}{$\begin{array}{l}\text { Heterogeneity. } \mathrm{Chi}^{2}=1.40, \mathrm{df}=1(\mathrm{P}=0.24) ; \mathrm{I}^{2}=28 \% \\
\text { Test for overall effect: } Z=3.97(P<0.0001)\end{array}$}} & & & \\
\hline & & & & & & & $\begin{array}{ll} \\
0.01 & 1 \\
\text { Favours [experim }\end{array}$ & 11 Favours [control] & 100 \\
\hline B & $B Q C+N$ & & MTX & & & Risk Ratio & & Ratio & \\
\hline Study or Subgroup & Events & Total & Events & Total & Weight & M-H, Fixed, 95\% Cl & $\mathrm{M}-\mathrm{H}$, & ed, $95 \% \mathrm{Cl}$ & \\
\hline Hongjian Wan 2012 & 6 & 26 & 7 & 26 & $6 \quad 50.0 \%$ & $0.86[0.33,2.21]$ & & & \\
\hline Li Rao 2016 & 4 & 72 & 7 & 72 & $250.0 \%$ & $0.57[0.17,1.87]$ & & & \\
\hline Total $(95 \% \mathrm{Cl})$ & & 98 & & 98 & $8 \quad 100.0 \%$ & $0.71[0.34,1.50]$ & & & \\
\hline Total events & 10 & & 14 & & & & & & \\
\hline \multicolumn{7}{|c|}{$\begin{array}{l}\text { Heterogeneity. } \mathrm{Chi}^{2}=0.28, \mathrm{df}=1(\mathrm{P}=0.60) ; \mathrm{I}^{2}=0 \% \\
\text { Test for overall effect: } Z=0.89(\mathrm{P}=0.37)\end{array}$} & $01 \frac{1}{0.1}$ & Favours [control] & 100 \\
\hline
\end{tabular}

Figure 11. Analysis of adverse effects of Biqi capsule or Biqi capsule plus MTX for the treatment of rheumatoid arthritis. (A) Adverse effects of Biqi capsule compared with MTX using a fixed-effects model. (B) Adverse effects of Biqi capsule plus MTX compared with MTX using a fixed-effects model. The ${ }^{2}$ and P-value were regarded as criteria of heterogeneity. The blue squares indicate the relative risk and their $95 \% \mathrm{CI}$. The black diamonds indicate the pooled relative risk and 95\% CI. CI, confidence interval; BQC, Biqi capsule; MTX, methotrexate; M-H, Mantel-Haentzel; df, degrees of freedom.

variety of AEs, specifically abnormal liver function and white blood cell reduction. These AEs may affect patient compliance to a certain extent. By contrast, AEs did not occur with Biqi capsule treatment. However, MTX and Biqi capsule may also cause gastrointestinal discomfort, but the severity of that induced by Biqi capsule is lower than that of MTX. A significant difference in AEs was observed between Biqi capsule and MTX, but no difference was present between Biqi capsule plus MTX and MTX. Thus, Biqi capsule had fewer AEs than MTX, and combined therapy did not increasethe frequency and severity of the AEs.

Of note, all of the trials selected for inclusion in the present meta-analysis were highly bias. The biases of random sequence generation, allocation concealment, blinding of participants and personnel, and blinding of outcome assessment were high, which led to conflicting results in the analysis, and thus lowered the validity and reliability of each individual study and the results of the meta-analysis. In addition, various trials included only had a small number of patients. Compared with other DMARDs, MTX is considered to be the first-line drug for treating inflammatory arthritis. Although the best duration of MTX treatment for RA is 6 months, the time until the drug takes effect is only 4-12 weeks (32). Therefore, the duration of the trials included in the presentstudy was sufficient to observe efficiency, but a longer duration may be better for observing long-term efficiency and AEs. In addition, the most important criteria, including ACR20, ACR50 and ACR70, were not reported in any of the studies. For these reasons, the conclusionsare not sufficiently convincing. Thus, prospective, large-sample and long-term studies are required in the future. As MTX is considered to be the cornerstone of treatment for RA and the anchor drug in combination with other DMARDs or biological agents (4), clinical studies on Biqi capsule plus MTX compared with MTX plus leflunomidemay be performed in the future.

In conclusion, treatment with Biqi capsule plus MTX appears to have more benefits for RA patients than MTX alone. In addition, administration of Biqi capsule had fewer AEs than that of MTX, and Biqi capsule plus MTX does not increase the number of AEs. Therefore, Biqi capsule may be suitable 
for use. However, the trials selected for inclusion in the present meta-analysis have numerous methodological flaws and a high risk of bias, which makes the conclusions insufficiently reliable. As the results of the present study suggest beneficial effects of Biqi capsule, high-quality RCTs are recommended in the future.

\section{Acknowledgements}

Not applicable.

\section{Funding}

This study was supported by the Science and Technology project of Guangdong Province (grant no. 2016KT1269), the Natural Science Foundation of Guangdong Province (grant nos. 2015KT1753 and 2017KT1805) as well as the Science and Technology Project of Tianjin (grant no. 15YDLJSY00090).

\section{Availability of data and materials}

The analysed data sets generated during the study are available from the corresponding author on reasonable request.

\section{Authors' contributions}

$\mathrm{XMC}$ and JQW wrote the manuscript, researched the literature, selected the trials, extracted the data and assessed the risk of bias. QCH designed the study and modified the manuscript. JYZ performed the meta-analysis. JHP and MJW were major contributors in performing the meta-analysis. ZSH analysed the data. YLC analysed the data and was a major contributor in writing the manuscript. $\mathrm{XHH}$ was a major contributor in analysing the data. MJW was a major contributor in writing the manuscript. RYH modified the manuscript, searched literatures, selected trials and extracted data. All authors read and approved the final manuscript.

\section{Ethical approval and consent to participate}

Not applicable.

\section{Consent for publication}

Not applicable.

\section{Competing interests}

The authors declare that they have no competing interests.

\section{References}

1. Han BK, Kuzin I, Gaughan JP, Olsen NJ and Bottaro A: Baseline CXCL10 and CXCL13 levels are predictive biomarkers for tumor necrosis factor inhibitor therapy in patients with moderate to severe rheumatoid arthritis: A pilot, prospective study. Arthritis Res Ther 18: 93, 2016.

2. Taylor PC, Moore A, Vasilescu R, Alvir J and Tarallo M: A structured literature review of the burden of illness and unmet needs in patients with rheumatoid arthritis: A current perspective. Rheumatol Int 36: 685-695, 2016.

3. Kirwan JR and Boers M: Biological treatment in rheumatoid arthritis: When to stop? Lancet 383: 288-289, 2014.
4. Pincus T, Gibson KA and Castrejón I: Update on methotrexate as the anchor drug for rheumatoid arthritis. Bull Hosp Jt Dis (2013) 71 (Suppl 1): S9-S19, 2013.

5. Halilova KI, Brown EE, Morgan SL, Bridges SL Jr, Hwang MH, Arnett DK and Danila MI: Markers of treatment response to methotrexate in rheumatoid arthritis: Where do we stand? Int J Rheumatol 2012: 978396, 2012.

6. Schiff M and Bessette L: Evaluation of abatacept in biologic-naive patients with active rheumatoid arthritis. Clin Rheumatol 29: 583-591, 2010.

7. Emery P: Optimizing outcomes in patients with rheumatoid arthritis and an inadequate response to anti-TNF treatment. Rheumatology (Oxford) 51 (Suppl 5): v22-v30, 2012.

8. Eriksson JK, Karlsson JA, Bratt J, Petersson IF, van Vollenhoven RF, Ernestam S, Geborek P and Neovius M: Cost-effectiveness of infliximab versus conventional combination treatment in methotrexate-refractory early rheumatoid arthritis: 2-year results of the register-enriched randomised controlled SWEFOT trial. Ann Rheum Dis 74: 1094-1101, 2015.

9. Meissner B, Trivedi D, You M and Rosenblatt L: Switching of biologic disease modifying anti-rheumatic drugs in patients with rheumatoid arthritis in a real world setting. J Med Econ 17: 259-265, 2014.

10. He YT, Ou AH, Yang XB, Chen W, Fu LY, Lu AP, Yan XP, Feng XH, Su L, Song YJ, et al: Traditional Chinese medicine versus western medicine as used in China in the management of rheumatoid arthritis: A randomized, single-blind, 24-week study. Rheumatol Int 34: 1647-1655, 2014.

11. American College of Rheumatology Committee to Reevaluate Improvement Criteria: A proposed revision to the ACR20: The hybrid measure of American college of rheumatology response. Arthritis Rheum 57: 193-202, 2007.

12. Zhang DZ, Sun SG, Li W and Zheng FZ: Experimental study of processing semen strychni on the effect of anti-Inflammatory in adjuvant arthritis rats. China J Chin Med 30: 539-541, 2015 (In Chinese).

13. Feng QS, Wang GF, Wang QS, Gao LN and Cui YL: Anti-inflammatory activity comparison of aqueous extract with monomer components of Biqi capsule. Chin J Exp Tradit Med Formul 22: 89-93, 2016.

14. He XW, Fan XP, Zhong T and He Y: Research progress on new drug delivery system of brucine to treat rheumatoid arthritis. Chin Arch Tradit Chin Med 33: 2908-2911, 2015 (In Chinese).

15. Liu W: Clinical observation of Biqi capsule on rheumatoid arthritis. Tianjin J Transit Chin Med 21: 474-475, 2004 (In Chinese).

16. Gao J, Zeng Y, Yu F, Zhuang LN, Yang L and Liu CX: Full side and dismantlement study on anti-inflammatory and analgesic effect of Biqi capsule. Chin Tradit Herbal Drugs 40: 93-96, 2009 (In Chinese)

17. Liu W and Liu XY: Research the effect of Biqi capsule on human cartilage glycoprotein-39 in peripheral blood mononuclear cells of patients with rheumatoid arthritis. China J TCM Pharm 24: 391-392, 2009 (In Chinese).

18. Liu W, Wu YH, Liu XY, Shen W and Wang P: Protective effect of the drug-containing serum with Biqi capsule on articular chondrocytes in vitro. China J Tradit Chin Med Pharm 25: 1283-1286, 2010 (In Chinese).

19. Moher D, Liberati A, Tetzlaff J and Altman DG; PRISMA Group: Preferred reporting items for systematic reviews and meta-analyses: The PRISMA statement. J Clin Epidemiol 62: 1006-1012, 2009.

20. Arnett FC, Edworthy SM, Bloch DA, McShane DJ, Fries JF, Cooper NS, Healey LA, Kaplan SR, Liang MH, Luthra HS, et al: The American Rheumatism Association 1987 revised criteria for the classification of rheumatoid arthritis. Arthritis Rheum 31: 315-324, 1988.

21. Jadad AR, Moore RA, Carroll D, Jenkinson C, Reynolds DJ, Gavaghan DJ and McQuay HJ: Assessing the quality of reports of randomized clinical trials: Is blinding necessary? Control Clin Trials 17: 1-12, 1996.

22. Higgins JP, Altman DG, Gøtzsche PC, Jüni P, Moher D, Oxman AD, Savovic J, Schulz KF, Weeks L, Sterne JA, et al: The Cochrane Collaboration's tool for assessing risk of bias in randomised trials. BMJ 343: d5928, 2011.

23. Liu W, Zhang L, Zhou YL and Liu XY: Influence of Biqi capsule on cytokine in rheumatoid arthritis. Tianjin J Tradit Chin Med 22: 290-291, 2005.

24. Bai HT and Li J: Clinical observation on 50 cases of Biqi capsule for treatment of rheumatoid arthritis. China J Tradit Chin Med Pharm 24: 1489-1490, 2009 (In Chinese). 
25. Jie HY, Wu QF and Ding ZX: Clinical study of Biqi capsule combined with methotrexate for treatment of rheumatoid arthritis. Zhongguo Zhong Xi Yi Jie He Za Zhi 32: 195-198, 2012 (In Chinese).

26. Wan HJ, Liu J, Xu JY and Fang HY: The efficacy of Biqi capsules combined with low dose methotrexate in the treatment of rheumatoid arthritis. Chin J Biochem Pharm 33: 669-671, 2012.

27. Rao L, Shen CJ, Shi ZQ and Yang J: Effects of integrated traditional and western medicine on rheumatoid arthritis. China J Tradit Chin Med Pharm 31: 546-548, 2016 (In Chinese).

28. Wu Q, Ding Z, Lu Y, Sun D, Feng L, Jie H, Su X, Chen W and Tang H: Clinical study of Biqi capsule for treatment of rheumatism. In: Proceedings of the 8th National Conference on Rheumatic Diseases in Traditional Chinese Medicine and Western Medicine 104-109, 2010 (In Chinese).

29. Yun C: Treatment of rheumatoid arthritis with Biqi capsule in 36 cases. J Pract Tradit Chin Med 20: 509, 2004.
30. Xu YM, Rong XF, Tan HF and Peng FF: Effects of Chinese medicine Biqi capsule on the expressions of JAK/STAT signal pathways in CIA rats. Immunol J 31: 1072-1076, 2015.

31. Bian XQ and Liu W: Experimental study on pharmacodynamics of Biqi capsule for treatment of rheumatoid arthritis. China J Tradit Chin Med Pharm 21: 773-774, 2006.

32. Weinblatt ME, Maier AL, Fraser PA and Coblyn JS: Longterm prospective study of methotrexate in rheumatoid arthritis: Conclusion after 132 months of therapy. J Rheumatol 25: 238-242, 1998 .

(i) $\mathrm{E}$ This work is licensed under a Creative Commons Attribution-NonCommercial-NoDerivatives 4.0 International (CC BY-NC-ND 4.0) License. 